\title{
Mice Help Unravel Mystery of Spanish Flu Virulence
}

An international team of researchers has shed new light on one of the biggest secrets about one of history's most prolific killers, demonstrating that a single gene may be responsible for the Spanish Flu's lethality.

The influenza pandemic of 1918-1919, often referred to as the 'Spanish Flu', killed between 20 and 40 million people, and is often cited as the most devastating epidemic in recorded history. There is as yet no consensus view on what made this strain so deadly. In 1999, researchers sequenced the virus isolated from preserved victims, making it possible to study the functions of particular proteins. Using a technique called reverse genetics, a team led by Yoshihiro Kawaoka, of the University of Wisconsin (Madison), reconstructed the genes that encode for the virus' surface glycoproteins-hemagglutinin (HA) and neuraminidase (NA) - and inserted them into a modern flu virus.

Although influenza $A$ does not normally cause disease in mice, this recombinant virus produced lethal infections characterized by high levels of chemokines and cytokines, as well as severe hemorrhaging in the lungs (Nature, 7 October). Kawaoka's group determined that the 1918 strain's virulence was related to its HA protein; mice infected with recombinant virus lacking the NA gene still became ill.

Understanding what made the 1918 strain of influenza so deadly may help contain future outbreaks. As Kawaoka's group writes in Nature, once researchers have identified the properties that make the 1918 HA gene so dangerous, "it should be possible to devise effective control measures and to improve global surveillance networks for influenza viruses that pose the greatest threat to humans as well as other animal species."

$-T . S$.

by researchers at the U.S. Army Medical Research Institute of Infectious Diseases (USAMRIID), the Centers for Disease Control and Prevention (CDC), and Stanford University now seeks to fill this informational void by developing potential primate models.

In two articles from the Proceedings of the National Academy of Sciences, U.S.A. (19 October) this team shows that cynomolgus macaques, when treated with sufficiently high doses of specific variola strains, develop a potentially fatal disease resembling smallpox, similar to that observed in humans. The India 7124 and Harper strains-initially cultivated in the USSR and UK, respectively-generated lesions and rapidly progressing disease when introduced by aerosol or injection, though at doses considerably higher than are necessary to infect humans. The investigators anticipate the need to refine their system further, so that smaller doses can trigger disease progression and mortality that more closely mirror human pathology, but nonetheless managed to obtain informative preliminary microarray data from the infected macaques. Among other things, they gained insight into variola's modulation of host immune response and cytokine release, which is believed to be a primary cause of mortality.

USAMRIID investigator Peter Jahrling hopes the WHO will be sufficiently impressed by these findings to back further studies. "[We] should be selecting out for the monkey-tropic virus ... I want to go back and ask the question, if we get the virus out of the spleen or the lymph node of the dead monkey and use it as an inoculum for the next passage, can we get by with a much lower dose?" He adds that their initial microarray findings suggest possibilities for treatment strategies, and that a monkey-specific variola strain could greatly assist in the diagnosis of infection and testing of therapeutic agents. "Understanding the immunosuppression associated with this disease is going to go a long way toward understanding its nature," he tells Lab Animal. "[W]e're optimistic that model develoment has progressed to the point where the FDA will accept it as a surrogate for human disease, and we'll be able to demonstrate and license drugs on the basis of their animal efficacy."

$-M \cdot E$. 